\title{
e-Phaïstos
}

e-Phaïstos

Revue d'histoire des techniques / Journal of the history

of technology

VII-2 | 2019

La sauvegarde du patrimoine scientifique

\section{Quatre mille ans d'histoire du cuivre. Fragments d'une suite de rebonds, sous la direction de Michel Pernot}

\section{Florian Téreygeol}

\section{(2) OpenEdition}

\section{Journals}

Édition électronique

URL : http://journals.openedition.org/ephaistos/5049

DOI : 10.4000/ephaistos.5049

ISSN : 2552-0741

Éditeur

IHMC - Institut d'histoire moderne et contemporaine (UMR 8066)

Référence électronique

Florian Téreygeol, «Quatre mille ans d'histoire du cuivre. Fragments d'une suite de rebonds, sous la direction de Michel Pernot », e-Phaïstos [En ligne], VII-2 | 2019, mis en ligne le 15 octobre 2019, consulté le 24 septembre 2020. URL : http://journals.openedition.org/ephaistos/5049 ; DOI : https:// doi.org/10.4000/ephaistos.5049

Ce document a été généré automatiquement le 24 septembre 2020.

Tous droits réservés 


\title{
Quatre mille ans d'histoire du cuivre. Fragments d'une suite de rebonds, sous la direction de Michel Pernot
}

\author{
Florian Téreygeol
}

\section{RÉFÉRENCE}

Michel Pernot (dir.), Quatre mille ans d'histoire du cuivre. Fragments d'une suite de rebonds, Bordeaux, Presses Universitaires de Bordeaux, Ausonius éditions, 2017, 355p.

1 "Quatre mille ans d'histoire du cuivre ", si le titre peut faire peur, le sous-titre rassure tout en aiguisant la curiosité : "fragments d'une suite de rebonds». Michel Pernot, ancien directeur de recherche au CNRS, réunit dans cet ouvrage des Presses Universitaires de Bordeaux une série de textes ayant pour point commun le métal rouge et ses alliages. Il s'agit d'une collection d'articles qui n'ont pas la prétention de restituer une quelconque histoire du cuivre comme il est clairement énoncé dans l'introduction: "l'exhaustivité étant invraisemblable, il s'agit d'éclairer quelques points particuliers de l'histoire technique du cuivre et de ses principaux alliages » ( $\mathrm{p}$. 19). Nous ne sommes pas dans une vision encyclopédique et c'est heureux. Pourtant Michel Pernot prône l'antidisciplinarité (p. 20), notion intéressante qui s'affiche en opposition avec la trans / inter / pluri / disciplinarité, mais la définition n'apparaitra qu'à la lecture globale de l'ouvrage.

2 Michel Pernot touche également un point non moins important de l'étude des métallurgies anciennes : c'est un des rares domaines où la diachronie peut être assumée sans se perdre. Traverser les périodes est une chose, ici il s'agit également de traverser les genres en faisant appel aussi bien à des historiens, des archéologues, des physiciens qu'à des hommes de l'art. L'ouvrage s'ouvre ainsi sur une série de trois articles formant 
une introduction portant autant sur l'histoire que sur la physique du matériau. Les bases sont posées et les textes peuvent se dérouler chronologiquement. Ces treize contributions qui composent le corps de l'ouvrage sont inégalement distribuées : cinq pour une large Antiquité, deux pour les périodes médiévale et moderne et trois en histoire contemporaine. Cette vision historique est suivie par trois miscellanées ne contredisant pas la logique du titre. Enfin, deux articles font office de conclusion.

3 La surreprésentation de l'Antiquité au sens large ne doit pas surprendre ; elle n'est que le reflet de la réalité de la recherche. Michel Pernot ouvre cette séquence en développant une réflexion sur la maîtrise du geste technique et les questions liées aux chaînes opératoires. De très bonnes illustrations de son propos sont données par Ziad El Morr au chapitre suivant. Les contributions d'Anne Lehoërff puis d'Alexis Gorgues introduisent la question métallurgique dans les sphères du social et du savoir. L'un comme l'autre pointent la relation ambivalente qui réunit le cuivre et le fer, tant dans la production que pour l'usage. Ce questionnement sur les liens existant entre les métallurgies du fer et des métaux non-ferreux a structuré depuis longtemps la recherche en imposant une dichotomie. Pourtant, celle-ci ne semble plus aussi justifiée à mesure que nous engrangeons les résultats de fouilles récentes. L'interconnexion étroite de ces deux mondes que l'on a trop rapidement séparés se retrouve jusqu'à la période médiévale ${ }^{1}$.

4 Le Moyen-Âge est traité rapidement par Nicolas Thomas dans une vision consumériste. Son auteur essaye de couvrir plus de 1000 ans d'histoire dont il faut surtout retenir l'importance à donner à un réel croisement des sources et des méthodes. La contribution qui porte sur la période moderne est plus surprenante car il s'agit d'une étude de cas s'inscrivant dans le système d'échange entre l'Europe et les Amériques. Cette situation avait été également décrite par Matilla Tascon en $1958^{2}$ pour les Amériques Centrale et du Sud à propos du mercure. Elle inverse le schéma traditionnel qui veut que le métal fasse le trajet d'ouest en est, et non l'inverse. Bien sûr, dans la conception européenne, l'échange est déséquilibré au profit du vieux continent. Ce qui part d'Europe, le cuivre comme le mercure (mais également le fer), n'a pas la même valeur que ce qui arrive : l'or et l'argent.

5 Les textes sur le cuivre à la période contemporaine montrent une autre façon d'approcher le métal rouge. Les sources écrites deviennent abondantes et nous entrons dans une histoire quantifiée. La contribution d'Alexandre Fernandez pointe la polarisation de la production du cuivre en liaison avec les innovations techniques et les ressources minérales, nous amenant de Swansea jusque dans le désert d'Atacama. C'est un des rares moments où l'on perçoit l'importance des ressources géologiques, qu'il s'agisse de combustible ou de minerai. L'étude de cas de Marianne Lassalle, Corinne Arvieu et Florent Laroche vient illustrer le rôle du cuivre dans une innovation de premier plan : la maitrise de la pression. La contribution de Christophe Bouneau clôt ce tour d'horizon en propulsant le cuivre dans notre quotidien. Il montre la lutte du métal rouge face à l'aluminium et, finalement, sa rémanence à chaque nouvelle révolution. L'auteur se fait même le chantre de l'industrie nucléaire au travers d'une vision à très long terme de la gestion des déchets nucléaires pour laquelle le cuivre devrait jouer un rôle de premier plan, au moins en Suède.

6 À l'issue de ce survol chronologique du cuivre dans nos sociétés occidentales, trois articles offrent un regard décalé. Le premier est celui de l'industriel Jean-Marie Welter qui retrace l'histoire d'un procédé, le laminage, et de son outil. Il poursuit ainsi le sillon 
tracé par Charles Frémont en 1906 qui est à l'origine d'une série d'études sur des objets techniques ${ }^{3}$. Le second est porté par un homme de l'art, Jean Dubos, qui nous fait prendre pied dans une archéologie expérientielle plus qu'expérimentale. En France, c'est une approche rarement osée depuis les travaux de Philippe Andrieux ${ }^{4}$, mais qui est largement répandue dans le monde anglo-saxon. Enfin, la dernière contribution, portée par Valeria Bongiorno, Justine Vernet et Paolo Piccardo, nous projette dans un domaine connexe : la conservation du patrimoine. Ancrée dans le présent, elle prend pour objet la statuaire exceptionnelle du cimetière de Gênes et introduit la question technique des traitements de surface.

7 Bien que conclusives, les deux dernières contributions, celle d'Anne Françoise-Garçon comme celle de Michel Pernot, n'ont pas vocation à synthétiser les précédentes. La première offre une série de réflexions conceptuelles transpériodes. Dans la seconde, " pour ne pas conclure », Michel Pernot s'ouvre au travers d'un objet atypique, un pied romain pliable, sur la question de la mesure. Il intègre à juste titre la question de l'essai qui fait l'objet de nombreuses recherches ces dernières années 5 .

8 S'il fallait pointer des absences, il y a celles dont Michel Pernot se défend : les mines et surtout la transformation du minerai en métal. Pour produire du cuivre, passé le stade de la cueillette du métal natif, il a fallu apprendre précocement à collecter le minerai dans le sous-sol. Les travaux ne manquent pas depuis les études des mines protohistoriques de Cabrières ${ }^{6}$, en passant par celles du Pays Basque ${ }^{7}$, de la Lorraine ${ }^{8}$ et jusqu'aux recherches sur les mines modernes du Chili ${ }^{9}$. Comme l'a bien montré l'article d'Alexandre Fernandez, la ressource naturelle est au cœur de l'économie-Monde. Pour la métallurgie extractive, l'absence se justifie moins facilement d'autant que la nature des minerais traités conditionne celle des alliages produits qu'il s'agisse des cuivres arséniés de la protohistoire ou du traitement des minerais polymétalliques (cuivres argentifères, bournonite) des périodes historiques.

9 Plus ambigus est l'absence d'une réelle approche sur un objet particulier que l'on suit en filigrane dans plusieurs articles : la monnaie. Celle-ci est d'abord conçu comme un outil économique mais c'est également, d'abord, un objet technique dont la production en série doit être assurée autant que contrôlée. Et le cuivre, allié ou non, fait intrinsèquement partie des métaux monnayés: monnaie de cuivre bien sûr, mais également de bronze, d'orichalque, de billon, d'argent et d'or. Le cuivre est présent dans quasiment tous les types de numéraire (à l'exception du monnayage d'électrum pour les périodes hautes). Il est difficile d'ignorer les travaux réalisés au sein du centre Ernest Babelon, composante de l'Institut de Recherche sur les Archéomatériaux. Ce laboratoire s'est fait de longue date une spécialité de l'étude des monnayages en prônant toujours une réelle approche transdisciplinaire ${ }^{10}$. La monnaie y est regardée comme un outil économique mais aussi comme un objet technique ${ }^{11}$.

10 Pour les périodes médiévale et moderne, certes parent pauvre en France, il faut également considérer les travaux de nos collègues anglo-saxons ${ }^{12}$ et allemands ${ }^{13}$ mais aussi les recherches entreprises sur la renaissance des grands bronzes ${ }^{14}$ comme sur l' instrumentum médiéval en alliage cuivreux ${ }^{15}$.L'approche expérimentale se trouve confinée à une de ces facettes : celle qui confie la façon à l'homme de l'art. Il en est d'autres qu'il serait trop long de développer ici et qui rejoignent un débat ouvert depuis les travaux d'Ascher et de Coles $^{16}$.

11 Mais à ces quelques remarques, on rétorquera qu'il ne s'agit que de fragments. L'objectif de l'ouvrage, clairement énoncé dès l'introduction, est atteint : «il s'agit de 
faire se rencontrer des regards sur des périodes chronologiques qui ne se rencontrent pratiquement jamais ". Ce recueil se pose comme une série de fenêtres ou chacun, en fonction de sa spécialisation, trouvera la matière à une ouverture toujours bienvenue.

\section{NOTES}

1. Voir les travaux récents de Catherine Verna et ceux de Philippe Braunstein : VERNA Catherine, L'industrie au village. Essai de micro-histoire (Arles-sur-Tech, XIV et XV siècles), Paris, Les Belles Lettres, 2017.

2. MATILLA TASCON Antonio, Historia de las minas de Almaden, Desde la epoca romana hasta el ano 1645, Madrid, 1958, vol. 1, p. 207 et ss.

3. FREMONT Charles, "Origine du laminoir. Notes présentées à l'Académie des Sciences ", Revue de métallurgie, vol. 5, 1908, p. 500-508.

4. ANDRIEUX Philippe, «Couler le bronze comme il y a 4000 ans », Dossiers d'Archéologie, 46, 1980, p. $72-77$.

5. TEREYGOL Florent, THOMAS Nicolas, «La coupellation des alliages cuivre - argent : Approches expérimentales de l'essai d'argent par voie sèche ", Revue d'Archéomètrie, vol. 27, 2003, p. 171-181.

6. AMBERT Paul, «Les mines préhistoriques de Cabrières (Hérault) : quinze ans de recherches. Etat de la question ", Bulletin de la Société préhistorique française, T. 92/4, 1995, p. 499-508.

7. PIERRE Francis, «Les mines de cuivre et d'argent de la haute Moselle. Apparition et évolution des techniques de percement à la poudre noire, Le Thillot (Vosges)», Lotharingia, V, Nancy, 1993, p. 91-159.

8. PIERRE, 1993, op. cit.

9. SLOTTA Rainer, SCHNEPEL Inga, Schätze des Anden, chiles kupfer für die welt, DBM, 2011, $608 \mathrm{p}$.

10. BARRANDON Jean Noël, PICARD Olivier, Monnaies de bronze de Marseille. Analyse, classement, politique monétaire, Paris, Editions du CNRS, 2007 (Cahiers Ernest-Babelon 10).

11. MILLE Pierre, TEREYGEOL Florent., L'Atelier monétaire royal de La Rochelle, Rennes, Presses Universitaires de Rennes, 2011.

12. DUNGWORTH David, NICHOLAS Matthew, «Caldarium? An Antimony bronze used for medieval and post-medieval cast domestic vessels ", Historical Metallurgy, Vol. 38, part. 1, 2004, p. 10-23.

13. ASMUS Bastian, Medieval Copper Smelting in the Harz Mountains, Coll. Montanregion Harz, 10, DBM, 2012.

14. CASTELLE Manon, Les techniques de fabrication de la grande statuaire en bronze 1540-1660 en France, Thèse de $3^{\mathrm{e}}$ cycle, Université de Versailles-Saint-Quentin-en-Yvelines, 2016. 
15. THUAUDET Olivier, Les accessoires métalliques du vêtement et de la parure de corps en Provence du XI ${ }^{e}$ au XVI ${ }^{e}$ siècle. Archéologie, techniques et économie d'une industrie méconnue, Thèse de $3^{\mathrm{e}}$ cycle, Aix-Marseille Université, 2015.

16. ASCHER Robert, " Experimental archaeology », American Anthropologist, 63, 1961, p. 793-816

COLES John M., Archaeology by experiment, Londres, 1973.

\section{INDEX}

Mots-clés : histoire des techniques, archéologie, métallurgie, cuivre

Thèmes : Un ouvrage nous a appris

Keywords : history of technology, archeology, metallurgy, copper

\section{AUTEURS}

\section{FLORIAN TÉREYGEOL}

Archéologue au sein de l'Institut de Recherche sur les ArchéoMATériaux au CEA-Saclay (Laboratoire Archéomatériaux et Prévision de l'Altération, LMC IRAMAT UMR5060 CNRS et NIMBE UMR3685 CEA/CNRS IRAMAT-LAPA), Florian Téreygeol est spécialisé dans l'étude des systèmes de production des métaux non ferreux depuis la mine jusqu'à l'objet fini. Directeur de fouilles en France (mine de Melle, Castel-Minier etc.) et à l'étranger (Yémen, Bolivie, Tadjikistan), il supervise les activités de la plateforme CNRS d'archéologie expérimentale des Arts du feu. 